\title{
The relative frequency effect and S-R compatibility
}

\author{
HAROLD L. HAWKINS and BRUCE D. FRIEDIN \\ University of South Florida, Tampa, Fla. 33620
}

The influence of relative stimulus frequency on choice reaction time (RT) was studied in a task containing many: 1 S-R mappings and 2 levels of S-R compatibility. Choice RT increased with declining stimulus frequency, and the magnitude of this decline remained invariant across levels of compatibility. Variations in response relative frequency exerted no influence on performance under either level of compatibility. These results are consistent with a perceptual interpretation of the relative frequency effect.

It has characteristically been found in studies of choice RT that when stimuli and responses are assigned on a 1:1 basis, response latencies to individual $S-R$ alternatives increase with declines in the probability with which those alternatives are presented (Fitts, Peterson, \& Wolpe, 1963; Hawkins \& Underhill, 1971). This finding, the so-called relative frequency effect, has been interpreted in various ways. Some researchers (e.g., Bertelson \& Tisseyre, 1966; Hawkins \& Hosking, 1969) have viewed the effec", as a reflection of perceptual bias in which more frequently occurring stimuli are favored during the process of stimulus discrimination. Others (e.g., Dillon, 1966) see the effect as one reflecting a response bias where more frequently occurring responses are favored during response selection. Still others (e.g., Sanders, 1970) have proposed that the effect reflects the emergence within the experimental context of a relative frequency-dependent differential in the associative strength of specific $S-R$ pairs (S-R translation model).

By using a design in which several unequally likely stimuli are assigned to each of several unequally likely responses, Bertelson \& Tisseyre (1966) and Hawkins, Thomas, \& Drury (1970) have been able to show that choice $\mathrm{RT}$ is insensitive to variations in response relative frequency, per se, but is responsive to variations in stimulus relative frequency as manipulated within a given response category. These results are inconsistent with the response bias view, which implies that RT will vary with response relative frequency, but are fully consistent with both perceptual bias and S-R translation views. The purpose of the present investigation was to evaluate comparatively these latter two interpretations.

The design was a variation on that developed by Bertelson \& Tisseyre (1966). On each trial, $S$ was presented with one of four possible stimulus alternatives. Two of these required one vocal response, and the remaining two required another. The relative frequency of stimuli varied within a response category, and response relative frequency varied across categories. Each $S$ was tested under one of two levels of S-R compatibility ( familiarity). Under the high-compatibility condition, the vocal responses were highly associated in Ss' experience with the stimuli to which they were paired. Under the low-compatibility condition, stimuli and their respective responses had probably never been experienced in association with one another. If the relative frequency effect is due to perceptual bias, the magnitude of the effect should be uninfluenced by variations in $S-R$ compatibility, since the speed with which a stimulus can be discriminated is presumably independent of the familiarity of the association between that stimulus and its required response. On the other hand, if the relative frequency effect is due to frequency-dependent differences in the speed of $S-R$ translation produced within the experimental setting, it is to be expected that the magnitude of the effect will decline with increasing S-R compatibility. The argument here is reasonably straightforward: if $S-R$ associations have been previously well learned (i.e., are highly compatible), the speed with which such associations can be made should not be strongly influenced by differential practice (i.e., differential relative frequency) during the experiment. If, however, the required $S-R$ associations are new (i.e., low in compatibility), differential practice should have a relatively strong effect. This analysis can be further clarified by reference to the classic skill acquisition curve which illustrates that performance during the learning of skilled tasks characteristically increases as a negatively accelerated function of practice. That is, early in training (during a period of low compatibility), differences in practice exert a relatively large effect on performance, whereas later on (during periods of high S-R compatibility), differences in practice have relatively little influence on performance.

\section{SUBJECTS}

The Ss were 10 men and 6 women enrolled in an undergraduate course on research methods. All Ss had normal or normal corrected vision, and all were naive with respect to RT research. Course credit was given for participation.

STIMULI AND APPARATUS

Stimuli consisted of the uppercase letters $A$ and $S$ and the numerals 4 and 5 (Tactype No. 5418). The stimuli were presented singly for a duration of 1 sec on a uniform white background at a viewing distance of $43.18 \mathrm{~cm}$ through a Polymetric two-field mirror tachistoscope. Response latencies were registered by a Hunter Klockounter. A microphone was suspended about $2.54 \mathrm{~cm}$ in front of $S$ at mouth level. Vocalizations into the microphone closed a Marietta voice-activated relay, which, in turn, terminated the Klockounter.

\section{PROCEDURE}

All Ss were pretested on a 40-trial practice task in which a $2.54-\mathrm{cm}$ square color patch, either green $(p=$ 50) or orange, was presented on each trial. The vocal responses during practice were the common names of the colors. Based on performance on this task, Ss were blocked into slow ( $N$ $=8$ ) and fast responders. Half the Ss in each block were assigned on a random basis to one of two levels of compatibility. The task, under both levels of compatibility, was to vocalize one of two responses to each of four possible stimuli. Stimuli and responses were paired in 2:1 fashion. The two stimuli requiring one of the responses differed in relative frequency (.61 vs .13 ), and the relative frequencies of the two stimuli assigned the remaining response were both .13. Thus, there were high- (HH) and low- (LH) frequency stimuli assigned the high-frequency response and two low(LL) frequency stimuli assigned the low-frequency response. The vocal responses were "LETTER" and "DIGIT." A preliminary study, conducted on four Ss drawn from the same source as those used in the experiment proper, revealed that the vocalization of these two utterances closed the Marietta voice-activated relay with about the same latency.

All Ss were tested on a total of 240 trials during a single experimental session lasting about $1 \mathrm{~h}$ and occurring within 3 days of the practice session. During the experimental session, the identity of, the correct relative frequency of, and the response appropriate for each stimulus were made constantly available to $S$ on an 
Table 1

Mean Correct Reaction Time (in Msec) and Percent Incorrect Responses (in Parentheses) Across Levels of Compatibility S-R Frequencies and Trial Blocks

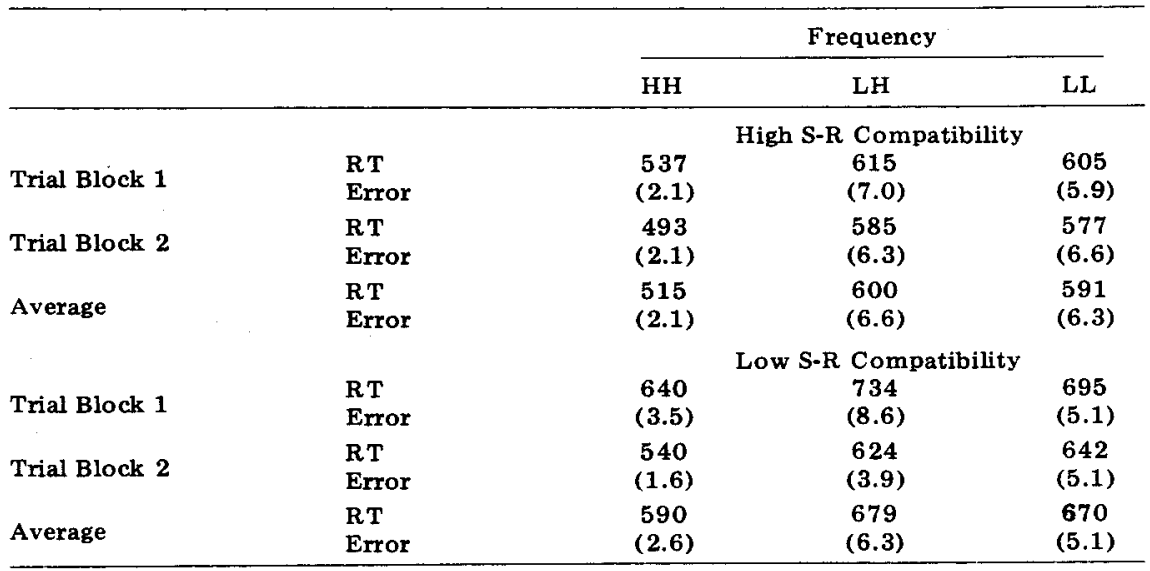

index card. The intertrial interval was $4 \mathrm{sec}$, with a warning tone of $1 \mathrm{sec}$ duration occurring immediately prior to stimulus onset. After each correct response, $S$ was informed of his $R T$ in milliseconds. Following each incorreci response, $\mathbf{S}$ was told that an error had been committed. Stimuli to which $\mathbf{S}$ incorrectly responded were not subsequently repeated. Short breaks were provided about every 24 trials.

High-Compatibility Condition

The eight Ss tested under the high-compatibility condition were required to vocalize the word "LETTER" when either A or S was presented and the word "DIGIT" when either 4 or 5 was presented. Each of the four stimuli served as the high-frequency alternative for two of the eight $\mathrm{Ss}$.

Low-Compatibility Condition

The eight Ss under this condition were required to vocalize the word "LETTER" when either 4 or 5 appeared and the word "DIGIT" when either $\mathrm{A}$ or $\mathrm{S}$ appeared. Again, each of the four stimuli served as the high-probability alternative for two of the eight Ss.

RESULTS AND DISCUSSION

Table 1 gives the mean latencies for correct responses (in milliseconds) and presents incorrect responses for the three $\mathrm{S}-\mathrm{R}$ frequencies $(\mathrm{HH}, \mathrm{LH}$, and LI), the two levels of compatibility, and the two levels of practice.

The latency data were subjected to a four-factor randomized blocks analysis in which premeasured speed (fast vs slow blocks) and compatibility level (high vs low) were treated as between-S variables and trial block (first vs last 120 trials) and S-R frequency were treated as within-S variables. This analysis revealed that latencies under the high-compatibility condition were significantly shorter th a $\mathrm{n}$ thos e under the low-compatibility condition, $F(1,12)=$ $6.21, \mathrm{p}<.05$, that performance improved across trial blocks, $F(1,12)=$ 20.97, $\mathrm{p}<.01$, and that latencies reliably differed across S-R frequencies, $\mathrm{F}(2,24)=17.70, \mathrm{p}<.01$. Fisher's least significant difference (LSD) test, with the criterion of significance set at .05 , indicated that the main effect of S-R frequency was due exclusively to an increase in latency between $\mathrm{HH}$ and $\mathrm{LH}$ stimuli $(\mathrm{LSD}=33 \mathrm{msec})$. The virtual identity of the latency increase between $\mathrm{HH}$ (89 $\mathrm{msec})$ and high- $(85 \mathrm{msec})$ compatibility conditions was reflected in the fact that the Compatibility by S-R Frequency interaction did not approach significance, $F(2,24)<1.0$.

These findings are consistent with the view that the relative frequency effect is produced by perceptual bias-a view which strongly asserts the independence of relative frequency and S-R compatibility. No support was obtained for the S-R translation interpretation which implies that the magnitude of the relative frequency compatibility. The present findings, and their implications, agree with those recently described by Hawkins \& Underhill (1971), who found that relative frequency and S-R compatibility were independent in both two- and four-choice tasks when 1:1 S-R correspondences were used. The findings are also in agreement and $\mathrm{LH}$ S-R frequencies across loweffect will decline with increasing $S-R$ with those of Bertelson \& Tisseyre (1966) and of Hawkins, Thomas, \& Drury (1970), who have shown that choice $R T$ is uninfluenced by response relative frequency per se. However, the results of the present study are clearly contrary to those of Sanders (1970), who reported a sizable interaction of the kind predicted from the S-R translation model. The cause of this seeming inconsistency could possibly relate to one or both of two procedural differences apparent across the two studies. First, the difference in difficulty (indexed by response latency) between high- and low-compatibility tasks was somewhat greater in the Sanders study. It must be emphasized, however, that this difference in difficulty is quite small (less than $40 \mathrm{msec}$ ) relative to the absolute magnitude of the interaction reported by Sanders. Second, Sanders used a four-choice task, whereas a simpler two-choice task was used in the present study. While one might be able to construct a plausible interpretation of the discrepancy between the two studies based on this difference, the interpretation would also have to explain why Hawkins \& Underhill (1971) found no Compatibility by Relative Frequency interaction in their four-choice task.

\section{REFERENCES}

BERTELSON, $P$ \& TISSEYRE, F Choice reaction time as a function of stimulus versus response relative frequency of occurrence. Nature, 1966, 212 , 1069-1070.

DILLON $P$ J Stimulus versus response decisions as determinants of the relative frequency effect in disjunctive reaction time performance. Journal of Experimental Psychology, 1966, 71, 321-330.

FITTS, P. M., PETERSON, J. R., \& WOLPE, G. Cognitive aspects of information processing: II. Adjustments to stimulus redundancy. Journal of Experimental Psychology, 1963, 65, 423-432.

HAWKINS, H. L., \& HOSKING, $K$. Stimulus probability as a determinant of discrete choice reaction time. Journal of Experimental Psychology, 1969, 82, 435-440.

HAWKINS, H. L., THOMAS, G. B., \& DRURY $K$. B. Perceptual versus response bias in discrete reaction time. Journal of Experimental Psychology, 1970, 84, 514-517.

HA WKINS, H. L., \& UNDERHILL, J. R. $S-R$ compatibility and the relative frequency effect in choice reaction time. Journal of Experimental Psychology, $1971,91,280-286$

SANDERS, A. F Some variables affecting the relation between relative stimulus frequency and choice reaction time. In $A$. F. Sanders (Ed.), Attention and performance III. Acta Psychologica, $1970,33,45-55$. 